\title{
A Brief Introduction to the Exposome and Human Health
}

\author{
Mark D Lucock* (1) \\ School of Environmental \& Life Sciences, University of Newcastle, Ourimbah, New South Wales, Australia
}

\begin{abstract}
The exposome refers to all environmental exposures (internal and specific/general external) that humans experience from conception through to death. This article examines the importance of both fundamental biological and public health perspectives within the context of the exposome, dealing with novel and well recognized concepts that include exposotype, issues of life stage/aging, and both short (epigenetic) and long-term (evolutionary) biological effects. The problem of scale, use of omics technology and database resources are also discussed. A special focus is also placed on the role of the ultraviolet light exposome in regard to the photolysis of folate and biosynthesis of vitamin D as important molecular mechanisms within human health and biology. Specifically, this includes critical nutrigenomic interactions. High-dimensional biology will permit extremely large scale initiatives in the future that will shed new light on the exposome by better characterizing a plethora of new and established biomarkers. However, smaller, delimited studies should not be ignored, as they can still help define aspects of the human exposome that remain unclear. Still, overall trends in the field are moving inexorably towards multiplexing metabolite and other omic analyses within large population studies. This article aims to reinforce the importance of exposomics via the idea, a fundamental tenet of biology, that human phenotype results from an amalgamation of genes and environment. What needs to be recognized is that phenotypes can be either adaptive or maladaptive. Within our phenome this process propagates disease or provides evolutionary advantage. In other words, the exposome is about more than simply public health.
\end{abstract}

Introduction

The exposome is a relatively new concept in health science that was initially laid out by Chris Wild in $2005 .{ }^{1}$ Few would deny that it represents a critically important field of study given the degree of negative anthropogenic change humanity is now subjected to. ${ }^{2}$ The exposome is broadly defined as "all environmental exposures that a person experiences from conception to death", ${ }^{3}$ and is thus an extraordinarily wide topic to get to grips with from a health perspective.

This enormous breadth poses significant conceptual and analytical problems to scientists. Initially, the concept was intended

Keywords: Exposome; Environment; Biomarkers; Vitamins; Epigenetics; Evolution. Abbreviations: CpG, 5'-cytosine-phosphate-guanine-3'; DNA, deoxyribonucleic acid; DOHaD, developmental origins of health and disease; DSSTox, distributed structure-searchable toxicity database; dTMP, thymidine monophosphate; HMDB, human metabolome database; HOX, homeobox; IGF2, insulin-like growth factor 2; IL10, interleukin 10; MPE, molecular pathological epidemiology; MTHFR, methylenetetrahydrofolate reductase; NTD, neural tube defect; SNP, single nucleotide polymorphism; UV, ultraviolet light; VDR, vitamin D receptor.

Received: November 26, 2020; Revised: December 9, 2020; Accepted: December 10, 2020

"Correspondence to: Mark D Lucock, School of Environmental \& Life Sciences, University of Newcastle, PO Box 127, Brush Rd, Ourimbah, New South Wales 2258, Australia. ORCID: http://orcid.org/0000-0002-0788-5177. Tel: +61-2-434-84109, Email: Mark.Lucock@newcastle.edu.au

How to cite this article: Lucock MD. A Brief Introduction to the Exposome and Human Health. Exploratory Research and Hypothesis in Medicine 2020;000(000):000000. doi: 10.14218/ERHM.2020.00070. to promote a wider assessment of exposure in epidemiological studies via the discovery of novel biomarkers that act as potential indicators of environmental influence on human health..$^{1,4-6}$ The term "environmental" specifically implies non-genetic factors, although there is a clear need for genomic data to be integrated with exposomal information to allow for the most comprehensive understanding of how environmental exposures impact human biology over the life span. Therefore, temporal factors are also critical, with exposures possible during pregnancy, infancy, childhood, adolescence, adulthood and in old age.

To facilitate a better understanding of the exposome concept, Wild $^{3}$ describes three non-genomic categories for evaluation: a) internal (endogenous), b) specific external and c) general external. These categories are fairly self-explanatory with internal exposomal factors encompassing hormones, oxidative challenges, inflammatory factors, allostatic load, microbiota, metabolism etc. Specific external factors are more varied and include electromagnetic radiation, pathogens, essential and non-essential dietary factors such as vitamins (indeed all food), environmental chemicals and pollutants, noise and a vast array of lifestyle factors such as tobacco, betel nut, alcohol, caffeine, pharmacologic agents, phytochemicals and other xenobiotic substances. The general external factors are broad and embrace social, economic and psychological influences. Something as simple as where you live (geography) might be critical if you live in a polluted city or in one at high latitude where low seasonal ultraviolet light (UV) levels limit vitamin $\mathrm{D}$ synthesis. These three non-genomic categories exhibit obvious overlap - allostatic load is a consequence of stress, but manifests 


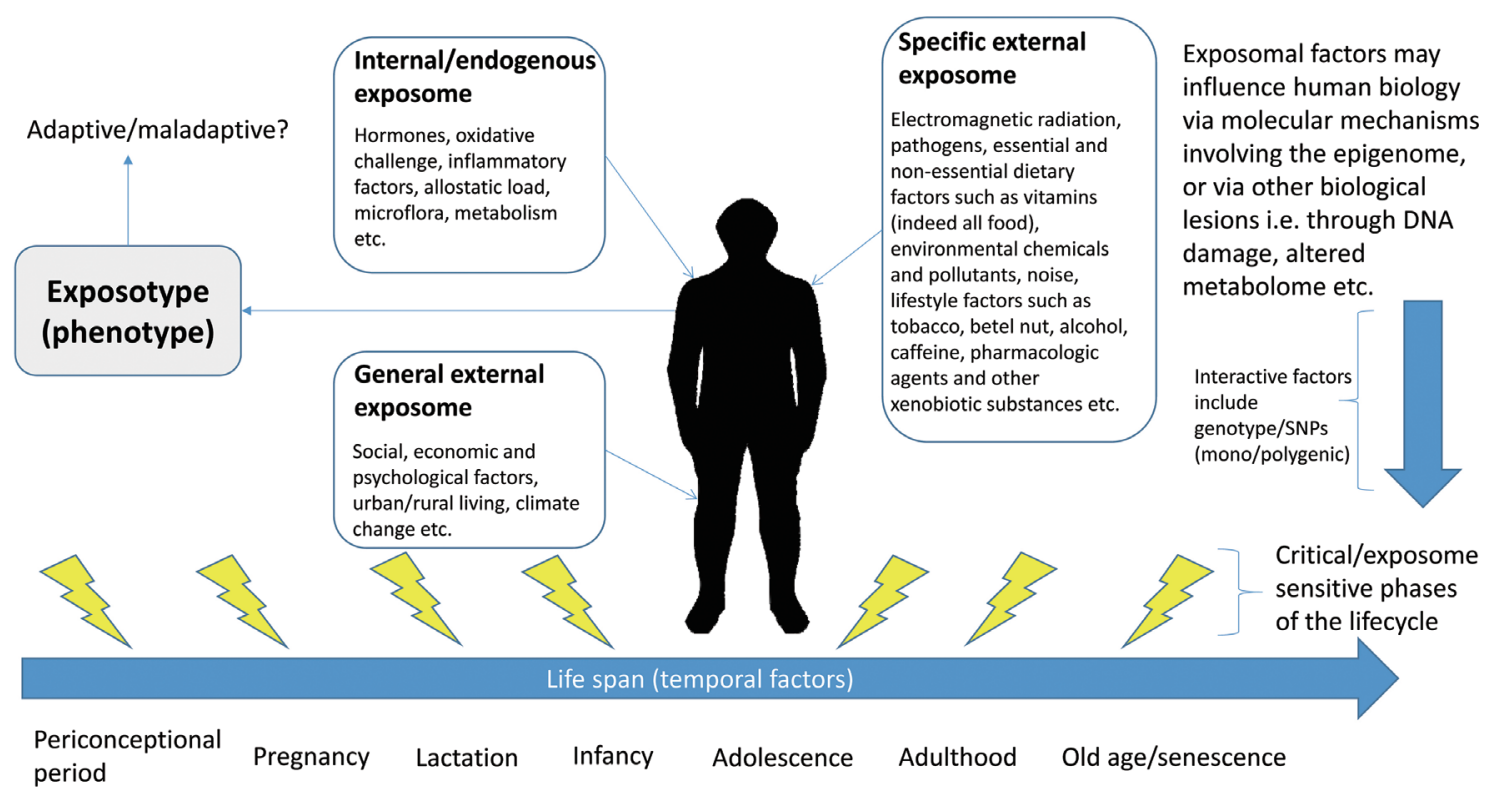

Fig. 1. Schematic showing some of the main exposomal factors that humans may experience. Attention is drawn to interactive factors such as gene variation and to selective vulnerable phases of the human lifecycle. Phenotypic outcomes such as exposotype may be maladaptive, resulting in negative health outcomes, but may also be adaptive within a longer term evolutionary timescale.

itself via metabolism, so categories a) and c) overlap. Anthropogenic climate change and its sequelae fit into all three categories $(a-c)$.

With such a complex topic as the exposome, this partitioning is quite useful in organising important aspects within a human biology perspective (see Fig. 1).

The field of exposomics has evolved quite rapidly over the past decade and a half, and reflects ever greater complexity given recent exponential advances in omics technologies. As a topic, the exposome is critically important, yet at the time of writing this article $\left(8^{\text {th }}\right.$ December 2020) a search of PubMed using the term "exposome" generated a mere 873 published articles. Despite this, recognition of the true importance of this field is emerging; a recent 2020 article in the journal Science states that given increased recognition of the dominant role that non-genetic factors play in disease, an effort to characterize the exposome at a scale comparable to that of the human genome is justified. ${ }^{7}$

\section{The problem of scale}

The exposome is easy to conceptualize at its broadest level, but complexity/depth of information make it challenging to take a holistic approach whereby exposomal components are seen as being intimately interconnected within a human biology paradigm that is only explicable by reference to the whole. It is far easier to focus on individual component details (a delimited system), rather than adopting holism. Having said that, the tools are now becoming available to help quantify the chemicals we are exposed to and the metabolic profiles that ensue. Genomics (particularly the role of common polymorphisms), epigenomics, proteomics, transcriptomics, pharmacogenomics, nutrigenomics, lipidomics, metabolomics and fluxomics are all omic tools that are now mature technologies, and are therefore poised to greatly influence this field as the age of bioinformatics and big data moves forward. An insightful, holistic view of the impact of different facets within the exposome will transpire, indeed, we are now looking at the application of highdimensional biology to environmental health modeling. ${ }^{8}$ Despite this, there are examples of simpler, delimited systems involving our exposome that are worthy of examination with respect to human biology, and which are elegant in their exposition (see below).

\section{From genotype and phenotype to exposotype}

High-dimension biology using a variety of omic technologies can now elucidate downstream biological changes that accrue and characterize the human exposure phenotype. This exposure phenotype derived from a specific exposure event has been referred to as an "exposotype". ${ }^{9}$ By blending genotype, proteomics and metabolomic data (along with other information such as life stage, etc). with detailed environmental exposure information, a route to understanding the molecular mechanisms that define specific exposotypes is possible. A good example of this would be that during the periconceptional period, a woman with elevated retinoids (vitamin A and/or synthetic analogues) due to treatment for skin complaints is more likely to experience a congenital malformation (neural crest/limb deformities) if she becomes pregnant. This mechanism is directly related to impaired $H O X$ gene function during embryogenesis. Exposure can also mean deficit, although this term usually implies excess. A shortage of folic acid during this same time frame leads to a neural tube defect (NTD)-affected pregnancy (i.e. spina bifida). It has been established that several genotypes can modify the occurrence and help define this exposotype. The best example is the possession of the 677TT-MTHFR genotype, which increases the risk of NTD, ${ }^{10}$ as do other factors such as exposure to certain pharmacologic agents, for example, valproic acid. ${ }^{11,12}$

Upstream processes that involve genes, transcription and proteins all contribute to the generation of metabolites. Being endproducts, metabolites are therefore perhaps closest to phenotype, and where concomitant xenobiotics are present, it becomes possible to begin defining exposotype. In fact, this underscores the 
science of metabolomics.

Potential systemic xenobiotics cover an enormous array of chemicals; from air pollutants to aflatoxins in food. However, as will be discussed in a later section, human exposure to physical factors such as temperature, UV light, day length, and season also influence human biology and help characterize exposotype.

\section{Cornucopia of data}

As might be expected, identifying a vast array of metabolites is challenging and is a rate-limiting factor to fully opening up this field. Fortunately, many databases are available for this purpose: The Human Metabolome Database (HMDB) is freely available and contains information about small molecule metabolites found in the human body (http://www.hmdb.ca/metabolites). The HMDB is intended to be used for applications in metabolomics, clinical chemistry, biomarker discovery and general education. It provides three kinds of data: 1) chemical data, 2) clinical data, and 3) molecular biology/biochemistry data. The database contains 114,260 metabolite entries. Four extra databases, namely DrugBank $(2,280$ drugs/metabolites), Toxic Exposome Database-toxin and toxin target database $(3,670$ toxins/environmental pollutants), small molecule pathway database $(25,000$ human metabolic disease pathways) and FooDB (28,000 food components/additives) are part of the HMDB suite. Many other high-quality resources are also available. The Distributed Structure-Searchable Toxicity (DSSTox) database provides a high-quality public chemistry resource for supporting improved predictive toxicology (https://www.epa. gov/chemical-research/distributed-structure-searchable-toxicitydsstox-database), while the Exposome-Explorer database provides details on biomarkers of exposure to environmental risk factors related to disease (http://exposome-explorer.iarc.fr/). One of the largest data repositories is PubChem (https://pubchem.ncbi.nlm. nih.gov/), which is an open chemistry database at the National Institutes of Health. It contains small molecules, but also larger ones (i.e. nucleotides, carbohydrates, lipids and peptides) and chemically modified macromolecules. PubChem acts as a repository of chemical structures, identifiers, chemical and physical properties, biological activities, patents, health, safety, toxicity data, and more. Another aspect adding to the complexity of this information involves recent advances in the human microbiome, a key internal exposomal factor. A relationship has been established between certain microbial signatures in the gastrointestinal tract and risk of diseases and maladapted phenotypes such as obesity. However, other dietary and biological factors are also thought to modify the microbiome, adding yet further complexity. ${ }^{13,14}$

While databases and tools do exist, there are the usual confounding issues at play. Such issues involve controlling for biological variance (diet, sex, body mass index, risk factors, exposure risk, temporal factors, gene-environment interactions, circadian rhythm, and the sheer number of known genomic single nucleotide polymorphisms [SNPs], etc.), as well as analytical variance (sampling time and storage, sample processing technique, lack of harmonization with respect to the type and use of instrumentation, and available compound library, etc.).

\section{Lifecycle considerations}

Early lifecycle events are critical to future health outcomes. The embryo and fetus are particularly vulnerable to environmental exposure. There are critical windows in utero where environmental stressors can disrupt or deflect the normal developmental trajectory, and hence "hard wire" changes in body structures, metabolism and physiology that can lead to a potential maladaptation to postpartum life in well nourished, developed societies. This has been shown to lead to chronic pathologies in later life. Indeed, this scenario is a major element within the Developmental Origins of Health and Disease $(\mathrm{DOHaD})$ paradigm. This paradigm is most often linked to prenatal diet, but evidence shows that air pollution and other environmental contaminants also impact fetal growth. ${ }^{15,16}$, with outcomes that influence individuals over their entire life span. ${ }^{17-19}$ It therefore seems likely that the "pregnancy exposome" is a critical starting point to life and hence an individual's future health prospects. ${ }^{19}$ By way of example, Tang and $\mathrm{Ho}^{20}$ examined epigenetic reprogramming and imprinting in the origin of disease, and discuss at length how early life environmental exposures can promote cancers.

The molecular mechanisms at play are many fold, but a critical factor is the maintenance of the methylome (epigenome). Gene $\mathrm{CpG}$ methylation profiles are critical to gene regulation and hence the provision of methyl groups is paramount. Dietary Methionine, methylfolate, vitamins $\mathrm{B}_{12}, \mathrm{~B}_{6}$ and $\mathrm{B}_{2}$, and choline are all critical in the provision of methyl groups, and any environmental exposure that modifies the availability of methyl groups is likely to have a significant impact. We now know that the provision of methyl groups is central to the $\mathrm{DOHaD}$. In addition, several gene mutations and environmental factors interact with the methylome and hence, conspire to play an important role in human development. ${ }^{21}$ It is important to note that environmental exposures can influence both germline and somatic cells via these types of molecular mechanisms.

Critical life stages extend beyond pregnancy and include lactation, infancy and late adulthood. All are subject to negative exposomal factors. Much has been written on this, particularly from a higher dimension perspective, so what follows are some thoughts on a simpler, yet important, delimited system, one that I have been fortunate enough to study for many years, and one that shifts perspective from more complex aspects of exposomal thinking.

\section{A novel example of a relatively delimited system}

The exposome is often discussed from a human health perspective, and this is fine. However, in truth it should also be considered from a human biology viewpoint. This is because environmental stressors can be adaptive as well as maladaptive. In that sense, they can contribute to evolutionarily adaptive phenotypes (one could argue a better term might be "adaptive exposotype"), and have acted over millennia to help shape human biology to the prevailing environmental conditions. Interestingly, the same environmental stressors can act over far shorter timescales (human lifespan) via their influence on epigenetic factors.

Both short-term human adaptation (epigenetic change) and long-term evolutionary change can occur due to variations in exposure to key vitamins. ${ }^{22,23}$ The food exposome represents the totality of dietary exposures, ${ }^{24}$ and is a substantial challenge to examine due to the wide range of foods consumed and the variability in the amount and frequency of intake, which are modulated by food preference, season, and other factors. ${ }^{24}$ Fortunately, two vitamins have such profound biological effects that their dietary abundance, and physicochemical loss (folate) or biosynthesis (vitamin D) through secondary environmental exposure (UV exposome) have shaped the human phenome from both evolutionary and health perspectives. Hence, these vitamins provide a good 
model for delimited exposome study.

Natural folate coenzymes are water-soluble vitamers, while D vitamers (i.e. 1, 25-dihydroxycholecalciferol/calcitriol) are fatsoluble, and are often considered to be more hormone than vitamin. Although these vitamins are very different at a chemical and biomolecular level, both these vitamin families participate in a common area of human biology. They are critical to the functional integrity of the genome and epigenome, and are also both responsive to the UV exposome, ${ }^{25}$ although their response to the UV exposome differs. Natural reduced folate vitamers such as 5-methyl- $\mathrm{H}_{4}$ folate are destroyed by $\mathrm{UV},{ }^{26}$ while vitamin $\mathrm{D}_{3}$ is photosynthesized in our skin via the action of UV. ${ }^{27,28}$ In the short-term, this can affect methyl group availability for epigenomic methylation, and thus regulated gene expression. ${ }^{21}$ As an example, the $\mathrm{CpG}$ methylation profile facilitates normal spermatogenesis and embryogenesis, with dysregulation linked to a number of clinically relevant phenotypes. As stated earlier, epigenetics is a fundamental mechanism within the $\mathrm{DOHaD}$ paradigm, ${ }^{29,30}$ a burgeoning subdiscipline that was born out of the Barker Hypothesis. ${ }^{31}$ Methylfolate is important because it supplies a pool of de novo methionine, which represents $50 \%$ of the total methionine requirement ${ }^{21}$; it follows that anything affecting the availability or stability of reduced folate coenzymes will alter the provision of methyl groups, with potential epigenetic consequences. A shortage of folate can also lead to restricted one-carbon unit flow into dTMP and hence DNA synthesis. This one-carbon shortage can lead to DNA fragility and may be important during embryogenesis. Therapeutically, it is also important in cancer occurrence where antifolate cancer treatments such as 5-fluorouracil block thymidylate synthase and restrict dTMP synthesis in rapidly growing cancer cells. A similar principle exists when using methotrexate as an abortifacient or as a chemotherapeutic agent for treating cancer. This drug blocks dihydrofolate reductase, an enzyme further upstream from dTMP synthesis, but one that still ultimately blocks one-carbon units for DNA synthesis. A simple dietary shortage of folate can also suppress erythropoiesis via impaired dTMP synthesis. One gene that influences the metabolic flow of folate derived one-carbon units is C677T-MTHFR. This SNP modifies the partitioning of carbon units towards de novo methionine for maintaining the methylome or dTMP for the fidelity of DNA synthesis, ${ }^{32}$ and shows the importance of genotype in folate-related cell survival and programming. Either of these processes may be relevant early in the lifecycle and in the occurrence and treatment of cancer. Early lifecycle changes to gene methylation profile have now been shown to extend into adulthood, and help substantiate the molecular basis of the DO$\mathrm{HaD}$, especially where methylation profile affects key genes. Prenatal exposure to famine (1944-45 Dutch Hunger Winter) led to less DNA methylation of imprinted insulin-like growth factor gene $(I G F 2)$ and increased methylation in leptin $(L E P)$, interleukin 10 (IL10) and other genes in famine subjects compared to unexposed same sex siblings 60 years after the famine exposome occurred. ${ }^{33}$ Lifecycle duration epigenetic changes in key metabolic regulatory genes indicate a mechanism through which very early life famine exposure influences adult metabolism and disease phenotype.

Vitamin D is also critical to the epigenome. The vitamin $\mathrm{D}_{3}$ activated vitamin D receptor (VDR) binds to 20,000 genome-wide sites within accessible chromatin. This epigenomic effect is significant as it modifies the transcriptome by activating or repressing vitamin $\mathrm{D}$ related target genes. Although vitamin $\mathrm{D}_{3}$ initiated VDR action potentially modulates the expression of many genes, it includes important chromatin modifiers and remodelers, and in this way, can influence the DNA methylation profile itself. ${ }^{34}$ Additionally, the gene encoding VDR is itself modulated by CpG methylation, with differential methylation in promotor regions control- ling gene expression. Critically, since vitamin $\mathrm{D}_{3}$ is a steroidal hormone, it acts like other hormones and is pivotal in promoting phenotypic plasticity, modifying gene expression and phenotypic outcomes in response to environmentally originated cues. Where those cues are unequivocally part of the human exposome, as with UV exposure, the consequences for human biology are significant. As with the Dutch Hunger Winter famine described above, it is also thought that early lifecycle UV exposure calibrates adult vitamin D metabolism, with evidence for a developmentally originated vitamin D homeostat that may alter related adult phenotypes (vitamin D metabolism, adult height and osteoporosis risk). ${ }^{35}$

While most health scientists tap into this concept from a disease/ wellness perspective, most likely within the DOHaD construct, far longer-term adaptive effects occur that reflect an evolutionary time scale. This is true for both folate and vitamin D.

An evolutionary multiplex of critical factors capable of adapting human skin phototype to an altering UV exposome seems likely to have occurred. ${ }^{36} \mathrm{UV}$ breakdown (photolysis) of folate in the skin is thought to have acted as a selection pressure for a darker skin phototype at tropical latitudes where human ancestors first evolved. Conversely, early humans migrating out of Africa likely required less skin pigmentation to allow for adequate vitamin D photosynthesis in the skin. The premise for this evolved trait revolves around the role of folate and vitamin $\mathrm{D}$ in providing a reproductive advantage, whereby natural selection operates on skin phototype to optimize folate stability and vitamin D synthesis according to the prevailing solar regime. ${ }^{37,38}$ Ultimately, the UV exposome-folatevitamin D multiplex interacts with a cassette of polymorphic genes that are known to be altered in the longer term to adapt the human skin phenome to the prevailing UV exposome..$^{22,23,26}$

\section{Future direction}

The magnitude of the exposome humans experience is so large that no single individual could ever embrace the entire discipline. Sources of pollution in the air, sea and fresh water are immense and variable; radiation can be overt as per the Chernobyl accident, or clinically important as in UV as a source of skin cancers that include melanoma. Although I give a single delimited example involving the UV wavelengths relevant to folate and vitamin D biology to show the scope of the field of exposome research, some of the best known environmental stressors include asbestos, heavy metals like lead and mercury, halogenated and polybrominated diphenyl ether (flame retardants), perfluorinated compounds (non-stick coatings etc), phthalates (plasticizers), formaldehyde and polyvinyl chloride (releases phthalates in use and dioxin when burnt). Many of the issues allied to the exposome have even broader implications in relation to, for example, climate change, and so the only way forward is to adopt a multidisciplinary approach. Expertise needs to focus on higher dimensional biology that utilizes the latest omic technologies, along with advanced data handling/ statistical analysis commensurate with the large pool of data generated. Of course, genetic variation can be included or excluded in studies, and this is quite acceptable where exposure-disease relationships are clearly established. However, from a prospective exploratory viewpoint, the more complete the analysis, the better understanding will be gained. Ultimately, as part of any future direction, research on the exposome should be linked to molecular pathological epidemiology (MPE) and tissue biomarker research. MPE is a relatively new integrated science that aims to better understand the interplay between etiological factors, cellular molecular characteristics, and disease evolution, and may help to address 
aspects of personalized medicine and disease prevention. ${ }^{39,40}$

An important point to make is that the exposome can relate to the unnaturally low presence of a substance as much as it can to elevated levels of a substance. This is particularly true when considering the food exposome. Also, in this context, foods are seasonal, and this seasonality can lead to variance in phytochemicals and important vitamins such as folate. This may have had historical significance (Ca. last 100 years), since it has been suggested that the seasonal cycle of abundance of folate-rich foods may have regulated embryo viability by acting as a selection factor for 677TMTHFR.$^{41}$ Ultimately, for exposomal research to flourish, harmonization across techniques and sampling is essential. Already, several large consortiums are emerging and the future seems assured given the backdrop of negative anthropogenic change we have to face up to, particularly from a public health perspective.

\section{Conclusions}

As a final thought, it is interesting to consider whether the current coronavirus disease (COVID-19) pandemic has altered the human exposome. Humanity has been exposed to more indoor time, increased levels of stress, and altered dietary habits. Although likely short term, it is interesting to speculate whether there is any relevance, particularly within a DoHAD framework.

\section{Acknowledgments}

None.

\section{Funding}

None.

\section{Conflict of interest}

No conflicts of interest exist.

\section{References}

[1] Wild CP. Complementing the genome with an "exposome": the outstanding challenge of environmental exposure measurement in molecular epidemiology. Cancer Epidemiol Biomarkers Prev 2005;14(8): 1847-1850. doi:10.1158/1055-9965.EPI-05-0456.

[2] Lucock M. The Anthropocene: Exploring its origins, biology and future. Am J Hum Biol 2020:e23476. doi:10.1002/ajhb.23476.

[3] Wild CP. The exposome: from concept to utility. Int J Epidemiol 2012;41(1):24-32. doi:10.1093/ije/dyr236.

[4] Wild CP. Environmental exposure measurement in cancer epidemiology. Mutagenesis 2009;24(2):117-125. doi:10.1093/mutage/gen061.

[5] Wild CP. Future research perspectives on environment and health: the requirement for a more expansive concept of translational cancer research. Environ Health 2011;10(1):S15. doi:10.1186/1476069X-10-S1-S15.

[6] Rappaport SM, Smith MT. Environment and disease risks. Science 2010;330(6003):460-461. doi:10.1126/science.1192603.

[7] Vermeulen R, Schymanski EL, Barabási AL, Miller GW. The exposome and health: Where chemistry meets biology. Science 2020;367(6476): 392-396. doi:10.1126/science.aay3164.

[8] Rattray JW, Deziel NC, Wallach JD, Khan SA, Vasilou V, Ioannidis JPA, et al. Beyond genomics: understanding exposotypes through metabolomics. Human Genomics 2018;12(1):4. doi:10.1186/s40246-0180134-x.

[9] Rattray NJW, Charkoftaki G, Rattray Z, Hansen JE, Vasiliou V, Johnson $\mathrm{CH}$. Environmental influences in the etiology of colorectal cancer: the premise of metabolomics. Curr Pharmacol Rep 2017;3(3):114-125. doi:10.1007/s40495-017-0088-z.

[10] van der Put NM, Steegers-Theunissen RP, Frosst P, Trijbels FJ, Eskes TK, van den Heuvel LP, et al. Mutated methylenetetrahydrofolate reductase as a risk factor for spina bifida. Lancet 1995;346(8982):10701071. doi:10.1016/s0140-6736(95)91743-8.

[11] Nau H. Valproic acid-induced neural tube defects. Ciba Found Symp 1994;181:144-160. doi:10.1002/9780470514559.ch9.

[12] Ornoy A. Valproic acid in pregnancy: how much are we endangering the embryo and fetus? Reprod Toxicol 2009;28(1):1-10. doi:10.1016/ j.reprotox.2009.02.014.

[13] Turner A, Veysey M, Keely S, Scarlett C, Lucock M, Beckett EL. Interactions between Bitter Taste, Diet and Dysbiosis: Consequences for Appetite and Obesity. Nutrients 2018;10(10):1336. doi:10.3390/ nu10101336.

[14] Ogino S, Nowak JA, Hamada T, Milner DA Jr, Nishihara R. Insights into Pathogenic Interactions Among Environment, Host, and Tumor at the Crossroads of Molecular Pathology and Epidemiology. Annu Rev Pathol 2019;14:83-103. doi:10.1146/annurev-pathmechdis012418-012818.

[15] Pedersen M, Giorgis-Allemand L, Bernard C, Aguilera I, Andersen AM, Ballester $F$, et al. Ambient air pollution and low birthweight: a European cohort study (ESCAPE). Lancet Respir Med 2013;1(9):695-704. doi:10.1016/S2213-2600(13)70192-9.

[16] Wigle DT, Arbuckle TE, Turner MC, Bérubé A, Yang Q, Liu S, et al. Epidemiologic evidence of relationships between reproductive and child health outcomes and environmental chemical contaminants. J Toxicol Environ Health B Crit Rev 2008;11(5-6):373-517. doi:10.1080/ 10937400801921320.

[17] Godfrey KM, Gluckman PD, Hanson MA. Developmental origins of metabolic disease: life course and intergenerational perspectives. Trends Endocrinol Metab 2010;21(4):199-205. doi:10.1016/j. tem.2009.12.008.

[18] Van den Bergh BR. Developmental programming of early brain and behaviour development and mental health: a conceptual framework Dev Med Child Neurol 2011;53(Suppl 4):19-23. doi:10.1111/j.14698749.2011.04057.x.

[19] Robinson O, Vrijheild M. The pregnancy exposome. Curr Envir Health Rep 2015;2(2):204-213. doi:10.1007/s40572-015-0043-2.

[20] Tang WY, Ho SM. Epigenetic reprogramming and imprinting in origins of disease. Rev Endocr Metab Disord 2007;8(2):173-182. doi:10.1007/s11154-007-9042-4.

[21] Lucock M, Yates Z, Martin C, Choi JH, Beckett E, Boyd L, et al. Methylation diet and methyl group genetics in risk for adenomatous polyp occurrence. BBA Clin 2015;3:107-112. doi:10.1016/j.bbacli.2014.11. 005.

[22] Lucock MD. Folic acid: beyond metabolism. J Evid Based Complement Alternat Med 2011;16:102-113. doi:10.1177/1533210110392950.

[23] Lucock M, Jones P, Martin C, Beckett E, Yates Z, Furst J, et al. Vitamin D: Beyond Metabolism.. Vitamin D: Beyond Metabolism. J Evid Based Complementary Altern Med 2015;20(4):310-322. doi:10.1177/21565 87215580491.

[24] Scalbert A, Huybrechts I, Gunter MJ. The Food Exposome. In: Dagnino S, Macherone A. (ed.). Unravelling the Exposome. Springer; 2019. p. 217-245. doi:10.1007/978-3-319-89321-1 8.

[25] Jones P, Lucock M, Veysey M, Beckett E. The Vitamin D-Folate Hypothesis as an Evolutionary Model for Skin Pigmentation: An Update and Integration of Current Ideas. Nutrients 2018;10(5):554. doi:10.3390/ nu10050554.

[26] Lucock M, Beckett E, Martin C, Jones P, Furst J, Yates Z, et al. UV-associated decline in systemic folate: implications for human nutrigenetics, health, and evolutionary processes. Am J Hum Biol 2017;29(2): e22929. doi:10.1002/ajhb.22929.

[27] Holick MF. Sunlight and vitamin D for bone health and prevention of autoimmune diseases, cancers, and cardiovascular disease. Am J Clin Nutr 2004;80(6 Suppl):1678S-1688S. doi:10.1093/ajcn/80.6.1678S. 
[28] Martin CE, Veysey M, Yates ZR, Lucock M. Vitamin D: Genetics, Environment \& Health. J Food Nutr Disor 2014;3:5. doi:10.4172/23249323.1000155.

[29] Kim KC, Friso S, Choi SW. DNA methylation, an epigenetic mechanism connecting folate to healthy embryonic development and aging. J Nutr Biochem 2009;20(12):917-926. doi:10.1016/j.jnutbio.2009.06. 008.

[30] Clare CE, Brassington AH, Kwong WY, Sinclair KD. One-Carbon Metabolism: Linking Nutritional Biochemistry to Epigenetic Programming of Long-Term Development. Annu Rev Anim Biosci 2019;7:263-287. doi:10.1146/annurev-animal-020518-115206.

[31] Barker DJ. The origins of the developmental origins theory. J Intern Med 2007;261(5):412-417. doi:10.1111/j.1365-2796.2007.01809.x.

[32] Sohn KJ, Jang H, Campan M, Weisenberger DJ, Dickhout J, Wang YC, et al. The methylenetetrahydrofolate reductase C677T mutation induces cell-specific changes in genomic DNA methylation and uracil misincorporation: a possible molecular basis for the site-specific cancer risk modification. Int J Cancer 2009;124(9):1999-2005. doi:10.1002/ijc.24003.

[33] Heijmans BT,TobiEW,Stein AD, PutterH, BlauwGJ, SusserES, etal. Persistent epigenetic differences associated with prenatal exposure to famine in humans. PNAS 2008;105(44):17046-17049. doi:10.1073/pnas. 0806560105.

[34] Fetahu IS, Höbaus J, Kállay E. Vitamin D and the epigenome. Front Physiol 2014;5:164. doi:10.3389/fphys.2014.00164.

[35] Lucock M, Thota R, Garg M, Martin C, Jones P, Furst J, et al. Early life- cycle UV-exposure calibrates adult vitamin D metabolism: Evidence for a developmentally originated vitamin $D$ homeostat that may alter related adult phenotypes. Am J Hum Biol 2019;31(4):e23272. doi:10.1002/ajhb.23272.

[36] Jablonski NG, Chaplin G. Colloquium paper: human skin pigmentation as an adaptation to UV radiation. PNAS 2010(Suppl 2):89628968. doi:10.1073/pnas.0914628107.

[37] Jones P, Lucock M, Veysey M, Jablonski N, Chaplin G, Beckett E. Frequency of folate related polymorphisms varies by skin pigmentation. Am J Hum Biol 2018;30(2):e23079. doi:10.1002/ajhb.23079.

[38] Jablonski NG. The evolution of human skin and skin colour. Annu Rev Anthropol 2004;33:585-623. doi:10.1146/annurev.anthro.33.070 203.143955.

[39] Ogino S, Lochhead P, Chan AT, Nishihara R, Cho E, Wolpin BM, et al. Molecular pathological epidemiology of epigenetics: emerging integrative science to analyze environment, host, and disease. Mod Pathol 2013;26(4):465-484. doi:10.1038/modpathol.2012.214.

[40] Ogino S, Nishihara R, VanderWeele TJ, Wang M, Nishi A, Lochhead P, et al. Review Article: The Role of Molecular Pathological Epidemiology in the Study of Neoplastic and Non-neoplastic Diseases in the Era of Precision Medicine. Epidemiology 2016;27(4):602-611. doi:10.1097/ EDE.0000000000000471.

[41] Lucock M, Yates Z, Ng X, Veysey M, Blades B, Travers C, et al. Preliminary evidence for genetic selection of 677T-MTHFR by natural annual cycle of folate abundance. J Nutrigenet Nutrigenomics 2008;1(12):24-29. doi:10.1159/000109872. 